\title{
Differences in motility of human X- and Y-bearing spermatozoa
}

\author{
H. Goodall and A. M. Roberts \\ Department of Physics, Guy's Hospital Medical School, London SE1 9RT, U.K.
}

\begin{abstract}
Summary. Human Y-bearing spermatozoa, as identified by the quinacrine staining technique, were found to be significantly more motile than $\mathrm{X}$-bearing spermatozoa under laboratory conditions. This difference is consistent with current estimates of the difference in mean head DNA content.
\end{abstract}

It has often been surmised that male-determining Y-chromosome bearing spermatozoa may differ in some respects from female-determining $\mathrm{X}$-bearing spermatozoa. Although early attempts to separate the two kinds on the basis of supposed differences in mass, density or surface charge met with little apparent success (Beatty, 1970), the recently developed quinacrine technique for detecting the human Y chromosome in spermatozoa (Barlow \& Vosa, 1970; Sumner, Robinson \& Evans, 1971) now enables these possibilities to be investigated without the necessity for experiments involving insemination. Results have been obtained which suggest that $Y$ spermatozoa may be more motile than X spermatozoa (Rohde, Portsmann \& Dörner, 1973; Ericsson, Langevin \& Nishino, 1973), although the results of Ericsson et al. are not confirmed by others (Evans, Douglas \& Renton, 1975; Ross, Robinson \& Evans, 1975). We describe here an experiment, based on the quinacrine staining method, which was designed to detect possible differences in motility between the two types of spermatozoon. Motile spermatozoa were layered at the bottom of a vertical column of fluid and were then allowed to swim upwards into the medium. If $\mathrm{Y}$ spermatozoa are more motile then they should outnumber X spermatozoa in the upper regions of the column (Roberts, 1972). This system possesses two useful features; firstly, equilibrium is eventually reached in which the numbers of organisms at given heights show no further changes, which simplifies the experimental procedures involved, and secondly, the degree of separation obtained can be used to estimate the difference in motility between $\mathrm{X}$ and $\mathrm{Y}$ spermatozoa.

\section{Methods and results}

Semen was provided by volunteer donors and used within 2-3 hr. Each sample was diluted to $10 \mathrm{ml}$ with warmed Tyrode's solution ( $\mathrm{pH} \mathrm{7.8)}$ ) and centrifuged at $1000 \mathrm{~g}$ for $15 \mathrm{~min}$. The pellet was resuspended in $5 \%$ bovine serum albumin $(25 \% \mathrm{BSA}$ in Tyrode's solution (Sigma) diluted with Tyrode's solution to $5 \%$ final concentration), the final sperm concentration being about $5 \times 10^{7} \mathrm{ml}^{-1}$ as determined by haemocytometer. A portion of this suspension was set aside as a control. Experiments were performed on columns $18 \mathrm{~mm}$ in total height, each of which comprised a cylindrical portion of $21 \mathrm{~mm}$ diameter and $10 \mathrm{~mm}$ height and an upper conical portion of $8 \mathrm{~mm}$ height through the apex of which the contents of the column could be expelled. The columns consisted of Tyrode's solution stabilized with a BSA gradient, varying continuously from $0.2 \%$ at the top to $2.0 \%$ at the bottom, which was delivered from a small two-compartment gradient former. A suspension containing about $5 \times 10^{6}$ spermatozoa was introduced through a side port at the bottom of the column as a layer $300 \mu \mathrm{m}$ thick. After $1 \mathrm{hr}$, when spermatozoa had distributed themselves in the column, the piston which formed the base of the column was pushed slowly upwards and selected volumes of liquid were collected from the top. The dead space of the system (the conical portion) corresponded to the basal $3 \mathrm{~mm}$ of the column. No disturbance of the contents of the column could be seen provided expulsion was performed slowly. Experiments were conducted with dead spermatozoa (heat treated 
at $65^{\circ} \mathrm{C}$ for $15 \mathrm{~min}$ ) to check that nonmotile cells were not carried upwards either by convection or by mixing during the elution of fractions. No cells whatever could be detected in fractions under these conditions.

Spermatozoa from fractions were washed in Tyrode's solution and centrifuged directly onto a microscope slide $(1500 \mathrm{~g}$ for $15 \mathrm{~min}$ ) within a specially constructed cell designed to fit inside a $100 \mathrm{ml}$ MSE bucket. The control suspension was exposed to $5 \%$ BSA for $15 \mathrm{~min}$ and was then diluted with Tyrode's solution so that controls and fractions were matched as nearly as possible for exposure to BSA; this was done to obviate the possibility that spermatozoa treated with BSA, which tends to absorb quinacrine, did not stain differently from controls. The prepared slides were then air-dried and fixed for $30 \mathrm{~min}$ in $3: 1(\mathrm{v} / \mathrm{v})$ methanol:acetic acid mixture.

Fraction slides and their respective controls were immersed in a $0 \cdot 2 \%$ solution of a quinacrine variant* (Bayer) in 1:1 (v/v) methanol:citrate-phosphate buffer, $\mathrm{pH} \mathrm{6.8,} \mathrm{for} 15 \mathrm{~min}$, destained in $2 \% \mathrm{KCl}$ in buffer for $2 \mathrm{~min}$ and mounted in $\mathrm{pH} 6.8$ buffer using No. 0 coverslips sealed with rubber solution. Slides were coded by a person unconnected with the project and then viewed under incident illumination with a Leitz Orthoplan microscope $(\times 95$ oil immersion objective, total magnification $\times 1200 ; 200 \mathrm{~W}$ mercury lamp, $3 \mathrm{~mm}$ BG 12 and K530 filters). Slides were counted by two observers using mechanical tripmeters, the faces of which were obscured until the end of the count. Spermatozoa were categorized as having either none, one, or two or more fluorescent spots (F-bodies), the first two categories being classified as $\mathrm{X}$ - and $\mathrm{Y}$-bearing spermatozoa respectively. Because of the small numbers of spermatozoa on fraction and matched control slides (typically about 1 per 10 microscope fields for the upper fractions) it was found that the observer could not deduce the origin (i.e. control or fraction) of any particular slide. Because of the necessity to accumulate data from many experiments semen from the same two donors was used throughout.

The results of 21 experiments are shown in Table 1. The average percentage of spermatozoa containing one F-body is $3.7 \%$ greater in fractions than in controls. A paired $t$-test indicates that this difference is significant at the $2 \%$ level $(t=2.81$; d.f. $=20 ; 0.02>P>0.01)$. We therefore conclude that $\mathrm{Y}$ spermatozoa (as determined by the quinacrine technique) are significantly more motile than $\mathrm{X}$ spermatozoa.

\section{Interpretation of the results}

To infer from this result the magnitude of the difference in motility between $\mathrm{X}$ and $\mathrm{Y}$ spermatozoa we have utilized a general theory of micro-organism movement under gravity (Roberts, 1972). Motile spermatozoa placed in a vertical column of fluid eventually set up an equilibrium distribution in which the number of spermatozoa, $n$, found above height $h$ in the column is given by

$$
n=\mathbf{N} \exp (-h / \mathbf{S})
$$

where $\mathrm{N}$ is the total number of spermatozoa and $\mathrm{S}$ is the scale height of the distribution. The scale heights were estimated in our experiments from the numbers of spermatozoa recovered from the columns. $\mathbf{S}$ (which is a measure of progressive motility) increased slightly with height due to the spread in motility within the suspension, the best estimate for the mean value being $S=500 \pm 100 \mu \mathrm{m}$. If the difference in scale height between $X$ and $Y$ spermatozoa is $\Delta S$, the percentage of $Y$ spermatozoa (P) above a given height should increase linearly with height at a rate

$$
\frac{\mathrm{dP}}{\mathrm{d} h} \simeq 25 \frac{\Delta \mathrm{S}}{\mathrm{S}^{2}}
$$

No significant correlation could be detected in our experiments between percentage increase and fraction height. This is probably a consequence of the variability of the staining procedure and of the small change in F-body count over the usable height range of $3-5 \mathrm{~mm}$. Since the percentage difference in F-body count must be zero at $h=0$ (the whole sperm population being included in this case), and is expected to increase linearly with increasing height, the mean rate of increase in these experiments has been evaluated; the mean \pm S.E.M. value is $1.0 \pm 0.35 \% \mathrm{~mm}^{-1}$. Taking into account the proportion of $Y$ chromosomes which are not detected by the staining technique (the count on controls being

*Chloromethoxyacridylaminodiethylaminopropanol dihydrochloride. 
Table 1. Quinacrine analysis of human spermatozoa collected in fractions above various heights in columns at $25 \pm 1^{\circ} \mathrm{C}$ (there were no significant differences between the mean counts obtained by the two observers, and both sets of results were therefore combined)

\begin{tabular}{|c|c|c|c|c|c|}
\hline \multirow[b]{2}{*}{ Height (mm) } & \multicolumn{2}{|c|}{ Control } & \multicolumn{2}{|c|}{ Fraction } & \multirow{2}{*}{$\frac{\text { Difference }}{(\%)}$} \\
\hline & Count & $\%$ & Count & $\%$ & \\
\hline 3 & 591 & $43 \cdot 5$ & 577 & $47 \cdot 5$ & $4 \cdot 0$ \\
\hline 3 & 231 & 52 & 347 & $48 \cdot 4$ & -3.6 \\
\hline 3 & 317 & $42 \cdot 3$ & 370 & $49 \cdot 5$ & $7 \cdot 2$ \\
\hline 3 & 225 & $40 \cdot 5$ & 264 & 46 & $5 \cdot 5$ \\
\hline 3 & 401 & $36 \cdot 4$ & 401 & $41 \cdot 9$ & $5 \cdot 5$ \\
\hline 3 & 405 & 46.9 & 460 & $43 \cdot 3$ & -3.6 \\
\hline 3 & 303 & $41 \cdot 6$ & 183 & 47 & $5 \cdot 4$ \\
\hline 4 & 176 & $36 \cdot 9$ & 129 & $47 \cdot 3$ & $10 \cdot 4$ \\
\hline 4 & 164 & $47 \cdot 6$ & 181 & $40 \cdot 3$ & $-7 \cdot 3$ \\
\hline 4 & 120 & 34 & 118 & $39 \cdot 8$ & $5 \cdot 8$ \\
\hline 4 & 231 & 52 & 383 & 51 & $-1 \cdot 0$ \\
\hline 4 & 108 & 32 & 190 & 47 & $15 \cdot 0$ \\
\hline 4 & 134 & $38 \cdot 8$ & 82 & 50 & $11 \cdot 2$ \\
\hline 4 & 317 & 42.3 & 155 & 32.9 & -9.4 \\
\hline 4 & 157 & 43.9 & 69 & $43 \cdot 5$ & -0.4 \\
\hline 4 & 175 & $41 \cdot 1$ & 137 & 48.9 & $7 \cdot 8$ \\
\hline 4 & 405 & 46.9 & 489 & $49 \cdot 1$ & $2 \cdot 2$ \\
\hline 4 & 400 & $40 \cdot 5$ & 235 & $48 \cdot 5$ & $8 \cdot 0$ \\
\hline 5 & 231 & 52 & 293 & $55 \cdot 6$ & $3 \cdot 6$ \\
\hline 5 & 401 & $36 \cdot 4$ & 403 & $42 \cdot 4$ & 6.0 \\
\hline 5 & 405 & 46.9 & 400 & 53.0 & $6 \cdot 1$ \\
\hline Mean & 281 & $42 \cdot 6$ & 279 & $46 \cdot 3$ & $3 \cdot 7$ \\
\hline S.D. & 128 & $5 \cdot 8$ & 147 & $5 \cdot 1$ & $6 \cdot 1$ \\
\hline S.E.M. & 28 & $1 \cdot 3$ & 32 & $1 \cdot 1$ & $1 \cdot 3$ \\
\hline
\end{tabular}

only $42.6 \%$ compared with the anticipated $49-50 \%$, the best estimate of the rate of separation in these columns is $1.25 \pm 0.4 \% \mathrm{~mm}^{-1}$. Thus $(\Delta \mathrm{S} / \mathrm{S}) \sim 0.025$, and the average difference in scale heights between $\mathrm{X}$ and $\mathrm{Y}$ spermatozoa is about $2.5 \%$.

The tendency for $\mathrm{Y}$ spermatozoa to swim higher up the column must necessarily result in an excess of X spermatozoa at the bottom. The application of Equation 1 to $X$ and $Y$ spermatozoa, together with $(\Delta S / S) \sim 0.025$, indicates that $X$ spermatozoa will predominate in the bottom $500 \mu \mathrm{m}$ of the column with a maximum separation (at zero height) of about $2.4 \%$. The presence of nonmotile spermatozoa will tend to reduce the separation in this region of the column, however, and the detection of this small excess of $\mathrm{X}$ spermatozoa over such a small volume would be extremely difficult in view of the variability in the staining procedure.

The scale height itself is given by

$$
\mathrm{S}=u /(2 \mathrm{~B})
$$

where $u$ is the swimming velocity and $\mathrm{B}$ is the hydrodynamic orientation parameter which governs the rate at which spermatozoa orientate under gravity (Roberts, 1972). Assuming that a $4 \%$ difference in mean DNA content between X and Y spermatozoa (Sumner et al., 1971) produces a mean difference in head volume (or density) of about $4 \%$, the mean difference in $u$ should be somewhat less than $0.2 \%$ and the difference in B about $2 \%$ (Roberts, 1972). The measured separation is thus consistent with the expected difference in the parameter $B$.

In conclusion, these experiments demonstrate that human $\mathrm{Y}$-bearing spermatozoa (as categorized by the quinacrine technique) are more motile than X-bearing spermatozoa. Although the difference in swimming velocity appears to be negligible, motile $\mathrm{Y}$ spermatozoa are better able to remain in suspension against the sedimentating effects of gravity. This difference in motility is consistent with estimates of the difference in DNA content of the two types, and it is unnecessary to invoke any other 
differences between them to account for the results. Although the experiments throw no light on the question of whether $Y$ spermatozoa traverse the female reproductive tract more rapidly on average than X spermatozoa (Parkes, 1974), the condition for zero separation is that the efficiency of sperm transport is completely independent of head size. Whilst this might be true for passive movement of immotile spermatozoa it seems less likely to be so for motile spermatozoa, and further information on the nature of transport in the female reproductive tract is required before this possibility can be assessed.

We thank the Medical Research Council for support, Dr S. Leibowitz for the provision of microscope facilities, Mr D. Mutton for advice on staining procedures and Mr J. Dixon (Bayer U.K. Ltd) for quinacrine dye.

\section{References}

Barlow, P. \& VosA, C.G. (1970) The Y chromosome in human spermatozoa. Nature, Lond. 226, 961962.

Beatty, R.A. (1970) The genetics of the mammalian gamete. Biol. Rev. 45, 73-119.

Ericsson, R.J., LANGevin, C.N. \& Nishino, M. (1973) Isolation of fractions rich in human $\mathrm{Y}$ sperm. Nature, Lond. 246, 421-424.

Evans, J.M., Douglas, T.A. \& Renton, J.P. (1975) An attempt to separate fractions rich in human $Y$ sperm. Nature, Lond. 253, 352-354.

Parkes, A.S. (1974) Sexuality and reproduction. Persp. Biol. Med. 17, 399-410.
ROBERTS, A.M. (1972) Gravitational separation of $X$ and Y spermatozoa. Nature, Lond. 238, 223-225.

Rohde, W., Portsmann, T. \& Dörner, G. (1973) Migration of Y-bearing human spermatozoa in cervical mucus. J. Reprod. Fert. 33, 167-169.

Ross, A., Robinson, J.A. \& Evans, H.J. (1975) Failure to confirm a separation of $\mathrm{X}$ - and $\mathrm{Y}$-bearing human spermatozoa using bovine serum albumin gradients. Nature, Lond. 253, 354-355.

Sumner, A. T., Robinson, J.A. \& Evans, H.J. (1971) Distinguishing between $\mathrm{X}, \mathrm{Y}$ and $\mathrm{YY}$-bearing human spermatozoa by fluorescence and DNA content. Nature, New Biol. 229, 231-233.

Received 25 May 1976 